\title{
DFT Study of the Effect of Pressure on Framework Materials Including ZIF-8 and Inorganic Zeolites
}

\author{
Wenlin Chen, ${ }^{1}$ Nancy L. Ross, ${ }^{1}$ G. Neville Greaves ${ }^{2}$ \\ ${ }^{[1]}$ Department of Geosciences, Virginia Tech, Blacksburg, VA 24060, USA \\ ${ }^{[2]}$ Department of Physics, Aberystwyth University, Aberystwyth SY23 3BZ, UK
}

Despite forming the critical basis for technologies in separation, catalysis, nuclear waste containment and biomedical applications, the relationship between the structural topology of the microporous framework materials and energetics of their formation and stability remain poorly understood. In this study, microporous materials including ZIF-8 and inorganic zeolites are being investigated using molecular dynamics methods and density functional theory (DFT) to reveal the structural changes and possible phase transitions in these structures at high pressure. In order to detect if phase transitions occur, the changes in the volume of the simulation cell are recorded with increasing pressure. In addition, the reversibility of the transition are followed by de-pressurizing compressed structures. Molecular dynamics snapshots at different stages in compression and decompression are used to identify pressure-induced phase transitions, including pressure-induced amorphization, and their decompression to the crystalline starting point. The diffraction patterns, radial distribution functions, and vibrational density of states calculated from the simulations are compared with experimental data. DFT simulations of ZIF-8 at high pressure, for example, have provided insight into changes associated with pressure-induced amorphization involving a low density amorphous (LDA) phase and a high density amorphous (HDA) phase. 